\title{
Perseverance Rover and Its Search for Life On Mars
}

\author{
James L. Green \\ NASA Headquarters, Washington D.C. USA
}

\begin{abstract}
Mars orbiters, landers, and rovers have made extraordinary discoveries about the evolution of Mars and its potential for life. At this time, it is clear, that the potential of ancient life on Mars has increased based on several discoveries. There have been many observed signs of ancient liquid water: surface and underground. There are past geological environments on Mars that had reasonable potential to have preserved the evidence of life, had it existed. The detection of complex organics by Curiosity has increased the potential for preserving "fingerprints of life" that may be locked away in the rock record. These and other factors have led NASA, with the support of other space agencies, to mount a Mars sample return (MSR) campaign. The first mission of MSR is the Perseverance rover. Designed to core and store rock, soils, and atmospheric samples in sealed tubes for later return, Perseverance landed in Jezero crater in February 2021 near an extensive ancient river delta. Other missions will follow to bring these samples back to Earth for further analysis. In addition, Perseverance carried the Ingenuity helicopter as a technology demonstration which has been tremendously successful and may lead to other future ariel missions on the red planet.
\end{abstract}

Keywords: Mars, habitability, sample return

\section{Introduction}

Today there is a fantastic array of operating missions at the planet Mars that include: orbiters, landers, rovers, and a helicopter. The operating missions at Mars shown in Figure 1, are from several international space agencies and represent the greatest armada of simultaneous active scientific instruments ever to be deployed at a planet beyond the Earth. In addition to the NASA orbiters, Mars Odyssey, Mars Reconnaissance Orbiter, and the MAVEN spacecraft, the European Space Agency (ESA) manages Mars Express and Trace Gas Orbiter, the Indian Space Research Organization manages the Mars Orbiter Mission, the United Arab Emirates manages the HOPE mission, and the China National Space Agency (CNSA) manages the Tiawen-1 orbiter. On the surface, the operating missions include NASA's Curiosity rover, the InSight lander, the Perseverance rover, and the Ingenuity helicopter joined by the CNSA lander and Zhurong rover. The purpose of this paper is to set the stage for the upcoming missions to Mars that will return rock cores, soils, and atmospheric samples as a step toward understanding the evolution of Mars and determining if Mars may have harbored life in its past.

\section{Past Observations}

From the array of orbiting missions at Mars it has been determined that Mars was a blue planet early on in its history with more than two-thirds of its northern hemisphere under water, but over time it lost this ocean. Mars also lost its intrinsic dynamo field about 4 billion years ago. A magnetosphere is known to provide resilience against solar storms removing the outer layers of the atmosphere (Dong et al., 2020). There is compelling evidence that Mars has lost a significant amount of atmosphere to outer space due to the direct solar wind interaction with the ionosphere and upper atmosphere thereby disrupting the water cycle and allowing its ocean to evaporate without replenishment (Jakosky \& Edwards, 2018, Lillis et al., 2015).

Today, partial protection of the Martian atmosphere from the solar wind is provided by the strong, localized remnant crustal magnetic fields (Dong et al., 2015, Ma et al., 2014). Current monitoring of Mars shows an ongoing loss of key atmospheric elements, such as oxygen, due to solar short wavelength radiation (EUV and X-rays) and solar wind energy inputs (Brain et al., 2015). The current NASA rovers on Mars 


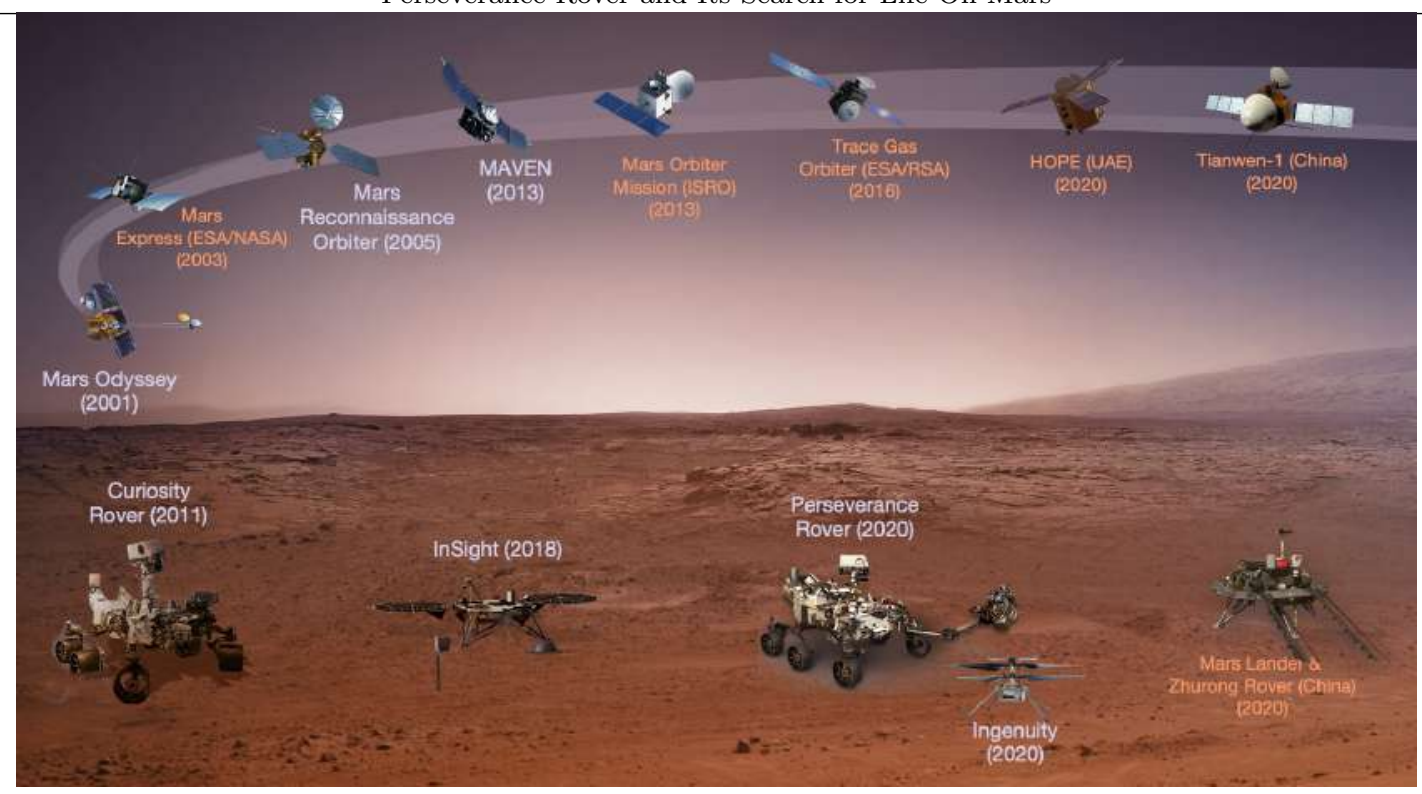

Figure 1. The international fleet of operating missions at Mars.

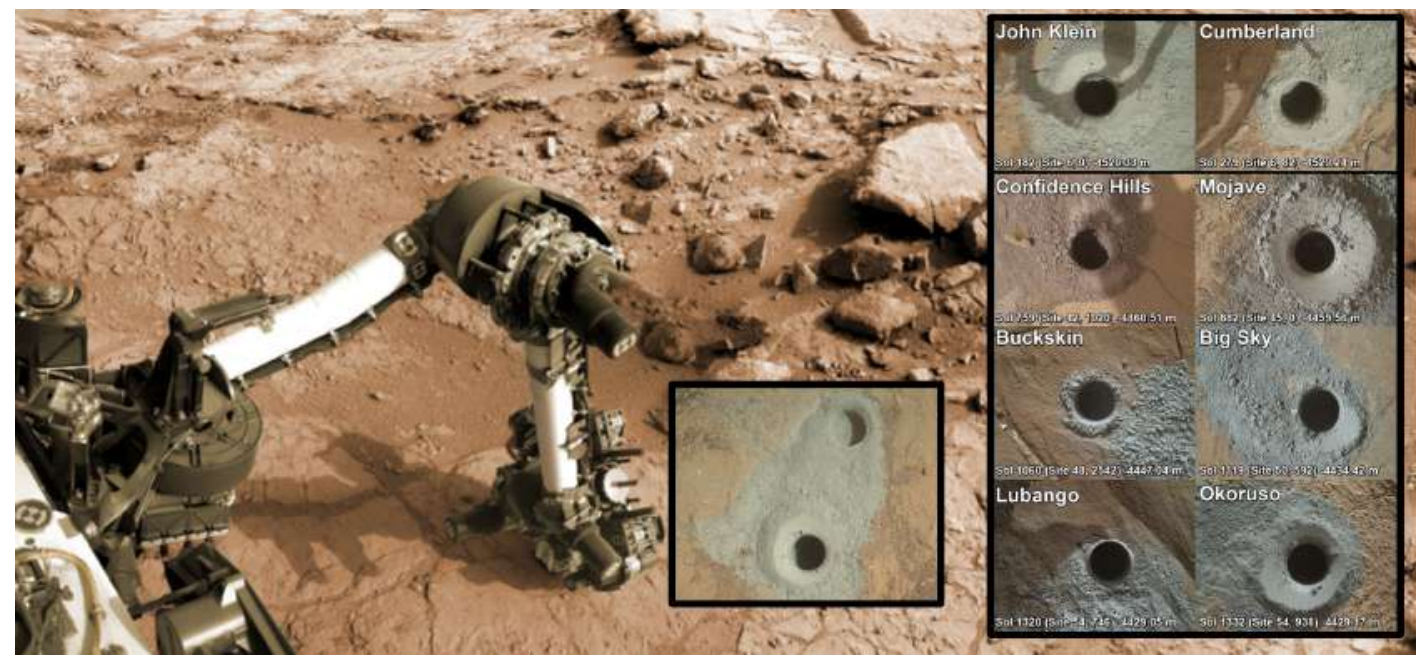

Figure 2. Mars subsurface material generated by the pulverizing drill on the Curiosity rover.

were designed to determine the habitability of past Mars by interrogating the soils and creating samples designed to be returned to Earth for future analysis.

The Curiosity rover, which landed in Gale Crater in August 2011, has uncovered a trove of important clues about Mars' distant past (Vasavada, 2015). For example, it is clear from the sedimentary material in Gale Crater that a significant amount of water filled the crater. Curiosity has a percussive drill allowing material to be pulverized for analysis. Drilling below the surface, about a centimeter to a centimeter and a half, the soils on Mars are very different than the oxidized surface. As shown in Figure 2, there's quite an array of different colored soils. Curiosity scoops up the pulverized soil material analyzing it in the Sample Analysis on Mars or SAM instrument. The key elements found in the soils by SAM include the chemical elements for life such as carbon, hydrogen, nitrogen, oxygen, phosphorus, and sulfur. The soils are moist, and they have nitrates.

Since Mars had a lot of water in its past, it must have had a significant atmosphere, and supported a significant greenhouse effect to keep the planetary surface warm to maintain a liquid ocean. Would it be also habitable for life at that time? Curiosity also found complex benzene and propane molecules as part of a larger macro molecule structure called kerogens (Eigenbrode et al., 2018). On Earth, typically organic constituents of kerogen are found in algae and woody plant products. These complex macro molecules tell us that the early Mars environment could have indeed been habitable. About 3 billion years ago, Mars went through a time of rapid climate change until it became the very dry and arid planet it is today. From this 


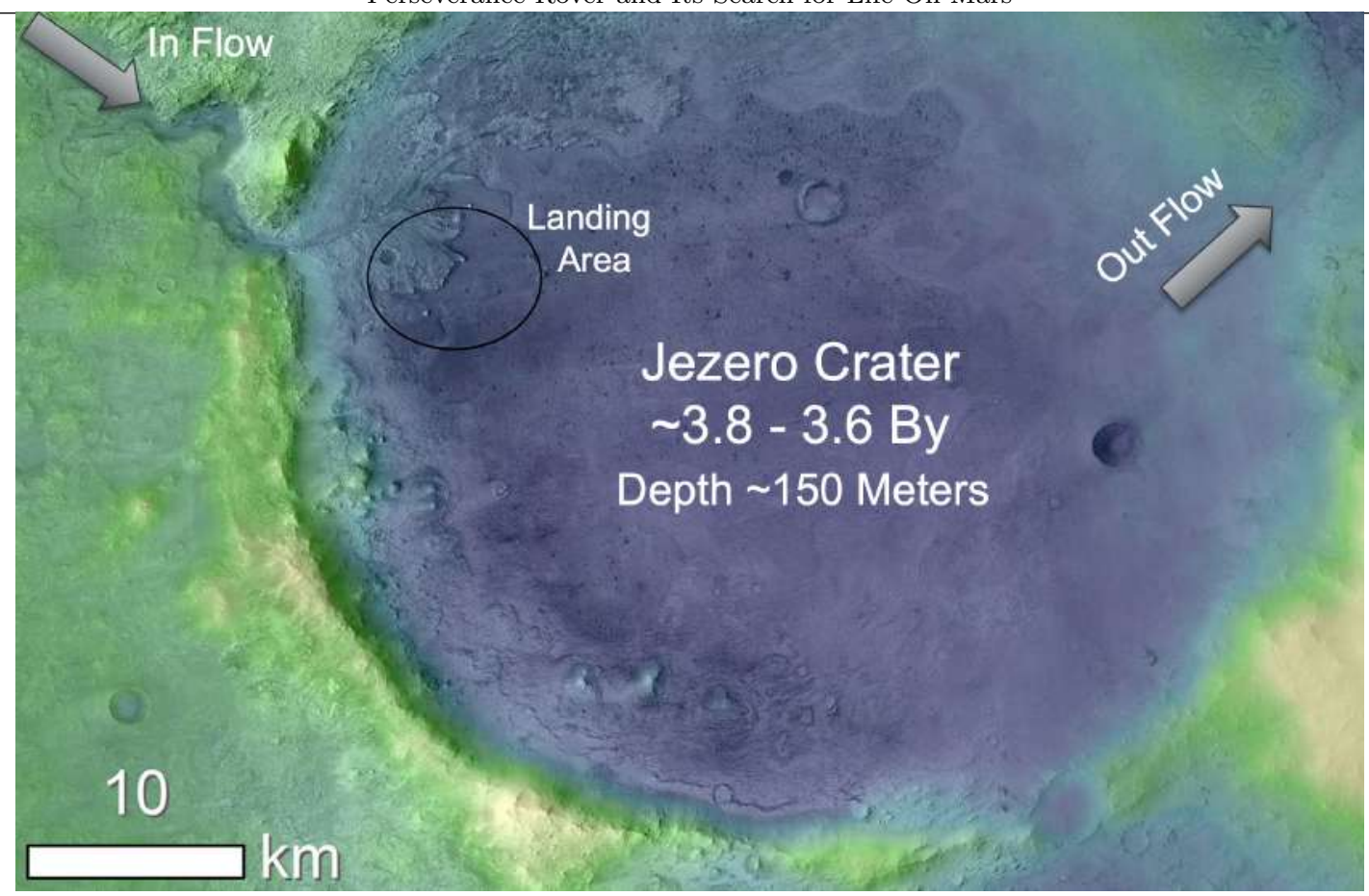

Figure 3. The Jezero crater and landing site of the Perseverance rover.

perspective, when we land our rovers on the surface of Mars today, the geology, the mineralogy, and its composition can be examined as if we are looking at early Mars. It is also important to note that about 3.6 billion years Earth life began. Could it also have begun on Mars and about the same time and if so, how can we find out? It is believed that a detailed rock record may hold the key to answering that question.

\section{Where are These Discoveries Leading Us?}

The recent Mars discoveries are leading us to conclude, that ancient life could have started on Mars, perhaps at the same time it started on Earth, because we've found the signs of past liquid water on its surface, strong indications of groundwater, and a past geological environment having a reasonable potential to preserve that evidence. A strong assumption in the past was that cosmic rays would break up organic material making it too difficult to find the macromolecules. But fortunately, we've been able to detect some of them. Now the detection of complex organics has increased the potential for finding, preserved in the soils and rocks, the "fingerprints of life." In summary, with our detailed knowledge of Mars, we now have a means to prioritize candidate sites and reason to believe that the evidence we are seeking may be preserved and that it is within reach of our exploration systems. The next logical step is for a Mars sample return (MSR) campaign to bring back the right samples.

\section{A River Delta in Jezero Crater: The Right Location}

About 4 billion years ago, the large ocean in the northern hemisphere on Mars was fed by a several rivers. At about 3.8 billion years a huge impact occurred on the ancient shoreline of this ocean creating a crater we named Jezero. The river flowed into the newly formed crater, as shown in the upper left corner of Figure 3, filling it up, until the water broke over its eastern crater wall (right corner of Figure 3) and into the Isidis Basin the lower extension of the ancient ocean. It is clear, as indicated from MRO images taken from orbit, that the rapid flowing river water upon meeting the rather still water within Jezero crater, dropped its sediments forming the extensive delta at this juncture we see today. The delta built up over time, with a diameter of approximately $10 \mathrm{~km}$ and a height of up to 65 meters.

Here on Earth, our own dried-up river delta's and ancient ocean shores preserve evidence of past life such as stromatolites that are 4 billion years old. Stromatolites are microbial mats that are built up over time in distinctive layers. Perhaps we will find similar types of signatures of life on Mars. On Earth there are over 5780 known minerals and about 337 of these are formed by the interactions with organic remains. 


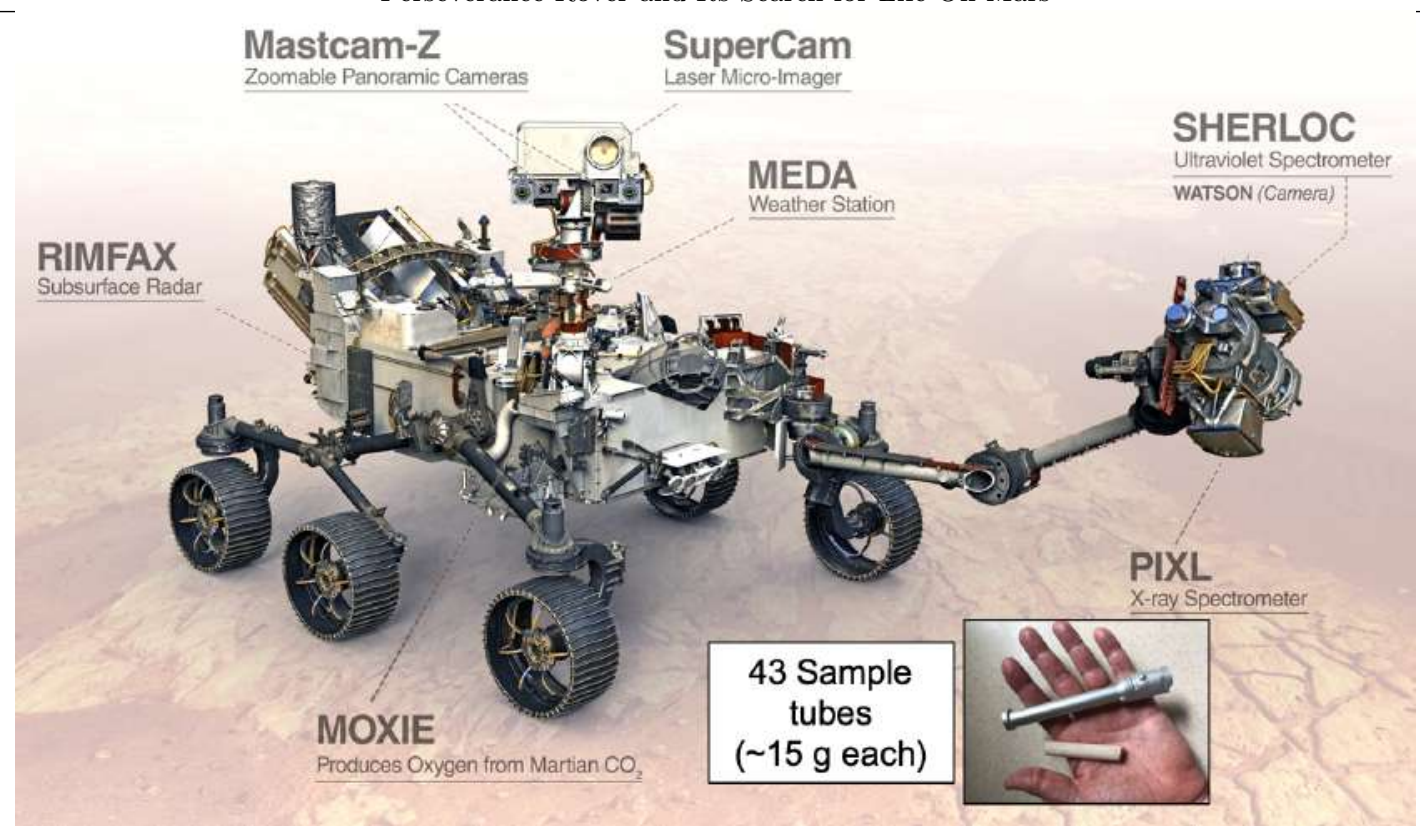

Figure 4. Perseverance rover instrumentation.

Once the Mars samples are returned to Earth for extensive analysis, not only will scientists be looking for the mineralogy as it may relate to the potential of past life, but also how the climate changed on Mars. It is believed that the climate change rapidly on Mars, but it is not known if that occurred over 10s of millions of years or several 100 million years.

After much deliberation, the landing site or ellipse for the Perseverance rover was determined to be just below the river delta in Jezero crater as shown in Figure 3. From orbit, MRO and Mars Express observed a variety of geological formations, not only the basaltic material from the adjacent volcano (west of the crater) thrown into the crater, but also olivine-carbonate bearing rock material. It will be up to Perseverance to validate these measurements, a process called ground-truth.

\section{Perseverance Rover: The Starting Point of MSR}

On February 18, 2021, NASA successfully landed the Perseverance rover while also carrying the Ingenuity helicopter, within the eastern portion of the landing ellipse on the Jezero crater floor just southeast and beyond the river delta. The purpose of NASA's Perseverance Rover is to create and cache critical samples for later return (Farley et al., 2020). Figure 4 is an overview of the instruments on the rover. Perseverance has many international instruments along with 23 cameras (9 engineering, 7 science, and 7 that were used during its entry, descent, and landing). The international instruments include: RIMFAX from Norway; SuperCam: NASA and France; MEDA from Spain; and a small retroreflector (not shown) from Italy. On the arm of the rover is a powerful drill designed to core rock, even as hard as granite, about the size of a chalkboard marker that is then placed into a metal tube producing a cache of samples. The rover has instruments that are designed to also provide a significant amount of contextual data about the geology, fine-scale mineralogy, chemistry, and atomic and molecular composition of targeted areas. This information is critical in determining where samples will be taken and how they may be interpreted once they have been returned to Earth and analyzed.

In addition to providing supporting sample information, Perseverance has the MEDA weather station (measuring temperature, pressure, wind velocity), a human exploration instrument, and a first ever Mars flying helicopter named Ingenuity. The average pressure of Mars is about $7 \mathrm{mb}$ and is composed of $95.9 \%$ CO2, $2 \%$ argon, $1.9 \%$ nitrogen, and several other trace gases. The Mars Oxygen In-situ Resource Utilization Experiment (MOXIE) is designed to demonstrate production of molecular oxygen (O2), which can be breathed by humans or used as rocket fuel, by electrochemically splitting the Mars carbon dioxide (CO2) atmosphere into carbon monoxide (CO) and O2. MOXIE has worked perfectly and can generate 6-10 grams of propellant grade $\mathrm{O} 2$ per hour from the Mars atmosphere.

Another technology demonstration that was successfully accomplished was the Ingenuity helicopter. To 
date (November 2021), Ingenuity has successfully flown its 5 test fights and 10 additional flights supporting Perseverance as an advance scout. Ingenuity has two cameras, one outward looking, and one downward looking and has flown to a maximum height of 10 meters and maximum distance of 271 meters. These flights and the demonstrated ability to easily maneuver in the thin Martian air indicates that flying technologies can be used successfully for future missions to the surface of Mars.

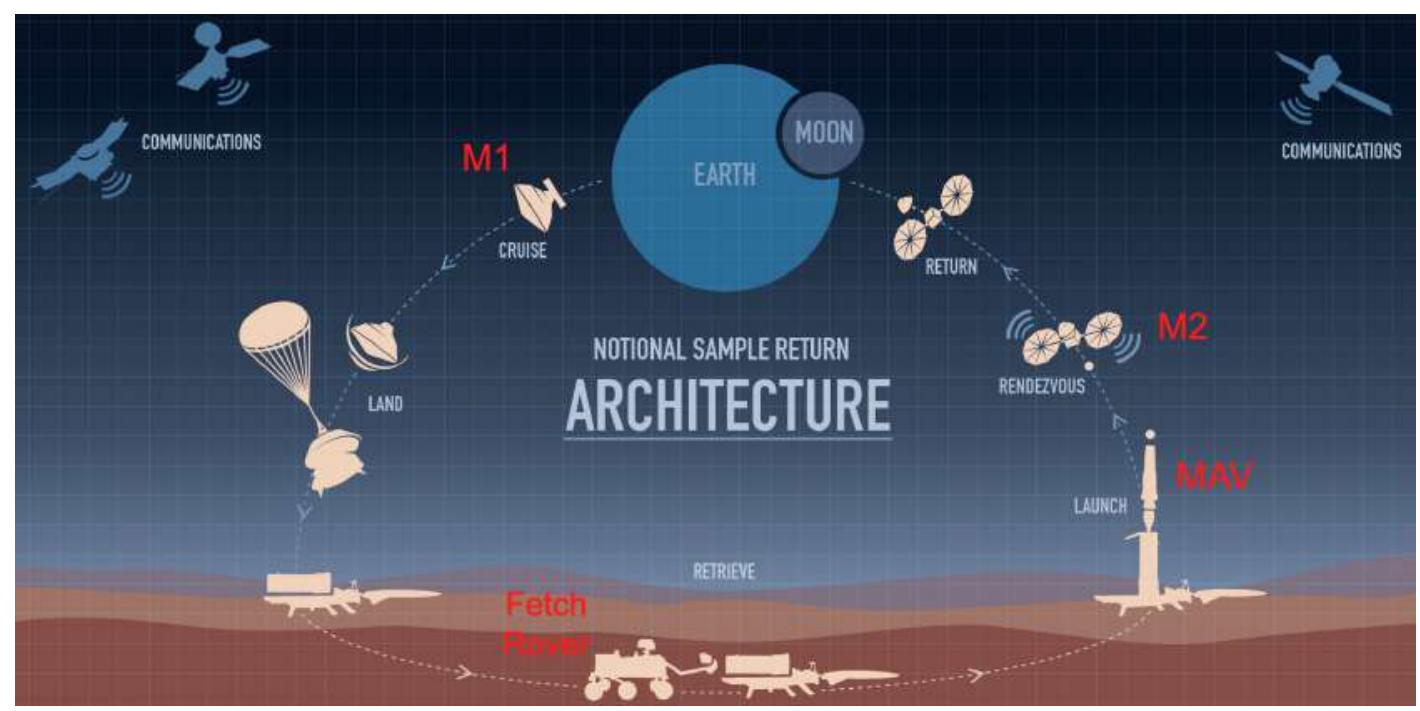

Figure 5. The notional Mars sample return architecture.

Perseverance has a total of 43 super clean sealed metallic sample tubes that can be filled over the next several years and will be placed on the surface in several piles for later pick-up and return. Currently (November 2021), Perseverance has already taken a total of 5 samples, which include 4 rock cores and one atmospheric sample. After exploring Jezero crater floor, Perseverance will climb up the delta, taking samples all along the way, depositing them in a few strategically placed stacks, and move into Nili Planum the high plains above the crater. Nili Planum gives Perseverance another opportunity at searching for signs of life in the rock record because it will be in an area of ancient hydrothermal systems and deep aquifers in the crust.

\section{The Sample Return Missions}

Shown in Figure 5 is the notional mission architecture for returning the cached samples which will start in 2026 and will consist of a series of missions from NASA and ESA. NASA's first mission (M1) will land a Mars Ascent Vehicle or MAV, along with a fetch rover. The rover will collect those samples, returning and loading them into the upper stage storage unit of the MAV. The MAV will then be erected and launched delivering the storage unit into low Mars orbit, where the second mission (M2), developed by ESA, will capture the sample storage unit and return it to the Earth. The sample storage unit will land in the desert of Utah in the United States and will immediately go into a biosafety facility to be curated, studied, and where the initial analysis will be completed. It is expected that at an appropriate time these samples will be released to the international science community for detailed analysis.

\section{Conclusion}

About 4 billion years ago, like the Earth, Mars was a blue planet, with a significant ocean and a thick atmosphere at the same time that life started on Earth. One of the challenges in searching for past life on Mars is that, while we know that the ancient surface had habitable conditions, we don't really know for how long and how rapidly it changed to the arid planet we see today. This situation calls into question whether Mars had time for life to spring up or not. The Perseverance rover has started the process of a sample return campaign that is designed to answer how the Red planet evolved over time and potentially, whether it harbored life in its past. 


\section{References}

Brain D. A., et al., 2015, Geophysical Research Letters, 42, 9142

Dong C., et al., 2015, Journal of Geophysical Research (Space Physics), 120, 7857

Dong C., Jin M., Lingam M., 2020, Astrophys. J. Lett. , 896, L24

Eigenbrode J. L., et al., 2018, Science, 360, 1096

Farley K. A., et al., 2020, Space Science Reviews, 216, 142

Jakosky B. M., Edwards C. S., 2018, Nature Astronomy, 2, 634

Lillis R. J., et al., 2015, Space Science Reviews, 195, 357

Ma Y., Fang X., Russell C. T., Nagy A. F., Toth G., Luhmann J. G., Brain D. A., Dong C., 2014, Geophysical Research Letters, 41, 6563

Vasavada A., 2015, EOS, March15, 16 